\title{
Effects of N-Linked Glycan on Lassa Virus Envelope Glycoprotein Cleavage, Infectivity, and Immune Response
}

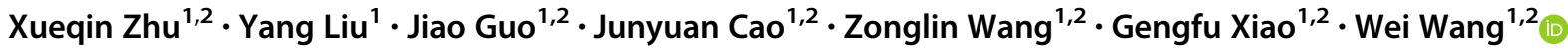

Received: 2 November 2020 / Accepted: 13 January 2021 / Published online: 10 March 2021

(c) Wuhan Institute of Virology, CAS 2021

\begin{abstract}
Lassa virus (LASV) belongs to the Mammarenavirus genus (family Arenaviridae) and causes severe hemorrhagic fever in humans. The glycoprotein complex (GPC) contains eleven N-linked glycans that play essential roles in GPC functionalities such as cleavage, transport, receptor recognition, epitope shielding, and immune response. We used three mutagenesis strategies (asparagine to glutamine, asparagine to alanine, and serine/tyrosine to alanine mutants) to abolish individual glycan chain on GPC and found that all the three strategies led to cleavage inefficiency on the 2nd (N89), 5th (N119), or 8th (N365) glycosylation motif. To evaluate $\mathrm{N}$ to Q mutagenesis for further research, it was found that deletion of the 2nd $(\mathrm{N} 89 \mathrm{Q})$ or 8 th $(\mathrm{N} 365 \mathrm{Q})$ glycan completely inhibited the transduction efficiency of pseudotyped particles. We further investigated the role of individual glycan on GPC-mediated immune response by DNA immunization of mice. Deletion of the individual 1st (N79Q), 3rd (N99Q), 5th (N119Q), or 6th (N167Q) glycan significantly enhanced the proportion of effector $\mathrm{CD}^{+}$cells, whereas deletion of the 1st (N79Q), 2nd (N89Q), 3rd (N99Q), 4th (N109Q), 5th (N119Q), 6th (N167Q), or 9th (N373Q) glycan enhanced the proportion of CD8 ${ }^{+}$effector T cells. Deletion of specific glycan improves the Th1-type immune response, and abolishment of glycan on GPC generally increases the antibody titer to the glycandeficient GPC. However, the antibodies from either the mutant or WT GPC-immunized mice show little neutralization effect on wild-type LASV. The glycan residues on GPC provide an immune shield for the virus, and thus represent a target for the design and development of a vaccine.
\end{abstract}

Keywords Lassa virus (LASV) · Glycoprotein complex (GPC) · N-linked glycan · Immune response

\section{Introduction}

Lassa virus (LASV) belongs to genus Mammarenavirus, family Arenaviridae. The natural reservoir of LASV is Mastomys natalensis in Africa, and humans are infected through direct contact with their excreta or exposure to the aerosol. Between 300,000 and 500,000 people are infected with LASV annually, and the mortality of hospitalized

Electronic supplementary material The online version of this article (https://doi.org/10.1007/s12250-021-00358-y) contains supplementary material, which is available to authorized users.

Wei Wang

wangwei@wh.iov.cn

1 State Key Laboratory of Virology, Wuhan Institute of Virology, Center for Biosafety Mega-Science, Chinese Academy of Sciences, Wuhan 430071, China

2 University of the Chinese Academy of Sciences, Beijing 100049, China patients ranges from 20\% to $70 \%$ (Houlihan and Behrens 2017). The United States Centers for Disease Control and Prevention classifies the virus as a Category A bioterrorism agent, and there is currently no drug or vaccine approved by the Food and Drug Administration capable of treating or preventing Lassa fever.

The envelope glycoprotein complex (GPC) of LASV is sequentially cleaved by signal peptidases and subtilisin kexin isozyme-1 (SKI-1)/site-1 protease (S1P) enzyme during the maturation process to obtain the stable signal peptide (SSP), receptor-binding GP1, and envelope fusion protein GP2. Mammarenavirus GPC is a heavily glycosylated protein. It has been estimated that N-linked glycosylation accounts for nearly $30 \%$ of the total mass of LASV GPC (Eichler et al. 2006; Hastie et al. 2017; Watanabe et al. 2018). The LASV lineage IV Josiah strain is the most commonly used strain in the development of LASV vaccines. It has $11 \mathrm{~N}$-glycosylation motifs (Asn-X-Thr/Ser, where $\mathrm{X}$ is any amino acid except proline) within the GPC 
(Fig. 1A). These $11 \mathrm{~N}$-linked glycans are distributed relatively evenly on the surface of the GPC in terms of spatial conformation, with seven glycans on GP1 and four on GP2. Glycans play critical roles in many biological functions associated with the GPC, such as cleavage, folding, receptor recognition, epitope shielding, and immune response (Sommerstein et al. 2015). Investigation on hospitalized patients infected with LASV found that a small amount of neutralizing antibodies could be detected in only a few convalescents at a relatively late stage of disease course, and that the neutralizing power of these antibodies was relatively low (McCormick et al. 1986; Johnson et al. 1987; Gunther et al. 2000; Sommerstein et al. 2015; Robinson et al. 2016). On the other hand, a previous study found that the cellular immune response plays a major role in immune defense against the LASV (ter Meulen et al. $2000,2004)$. We hypothesize that the large number of $\mathrm{N}$-linked glycans on the surface of LASV virions shield important epitopes within GPC, which adversely thereby making recovery from Lassa fever difficult. Therefore, studying the role of the $11 \mathrm{~N}$-sugar chains on the GPC of LASV with regards to the host immune response will help to identify the mechanism of immune escape employed by the virus and facilitate subsequent development of effective vaccines or therapeutic antibodies.

\section{Materials and Methods}

\section{Cells and Plasmids}

HEK 293 T, HeLa, and Vero cells were cultured in Dulbecco's modified Eagle's medium (HyClone, Logan, UT, USA) supplemented with $10 \%$ fetal bovine serum (FBS, Gibco, Grand Island, NY, USA). The pseudotype VSV bearing the GPC of LASV (strain Josiah, GenBank accession number HQ688673.1) as well as containing the Renilla luciferase (Rluc) reporter gene were generated as previously reported with the titer of $3 \times 10^{7} / \mathrm{mL}$ (Wang et al. 2018). The pseudotyped viruses carrying the WT and mutant GPC were also generated and treated with DNase I (Thermo Scientific, Waltham, MA, USA) and RNase A (ST 576, Beyotime, Shanghai, China) to remove the unpackaged DNA and RNA. The genome copies of the pseudotyped viruses were quantified using qPCR, and Vero

A

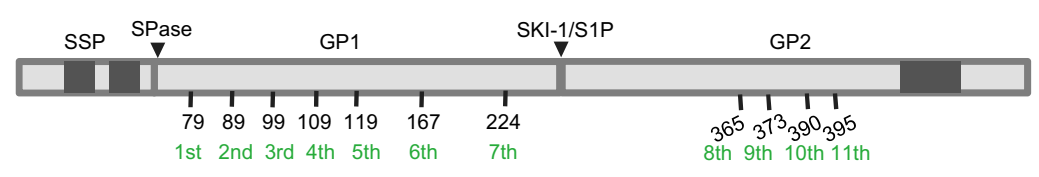
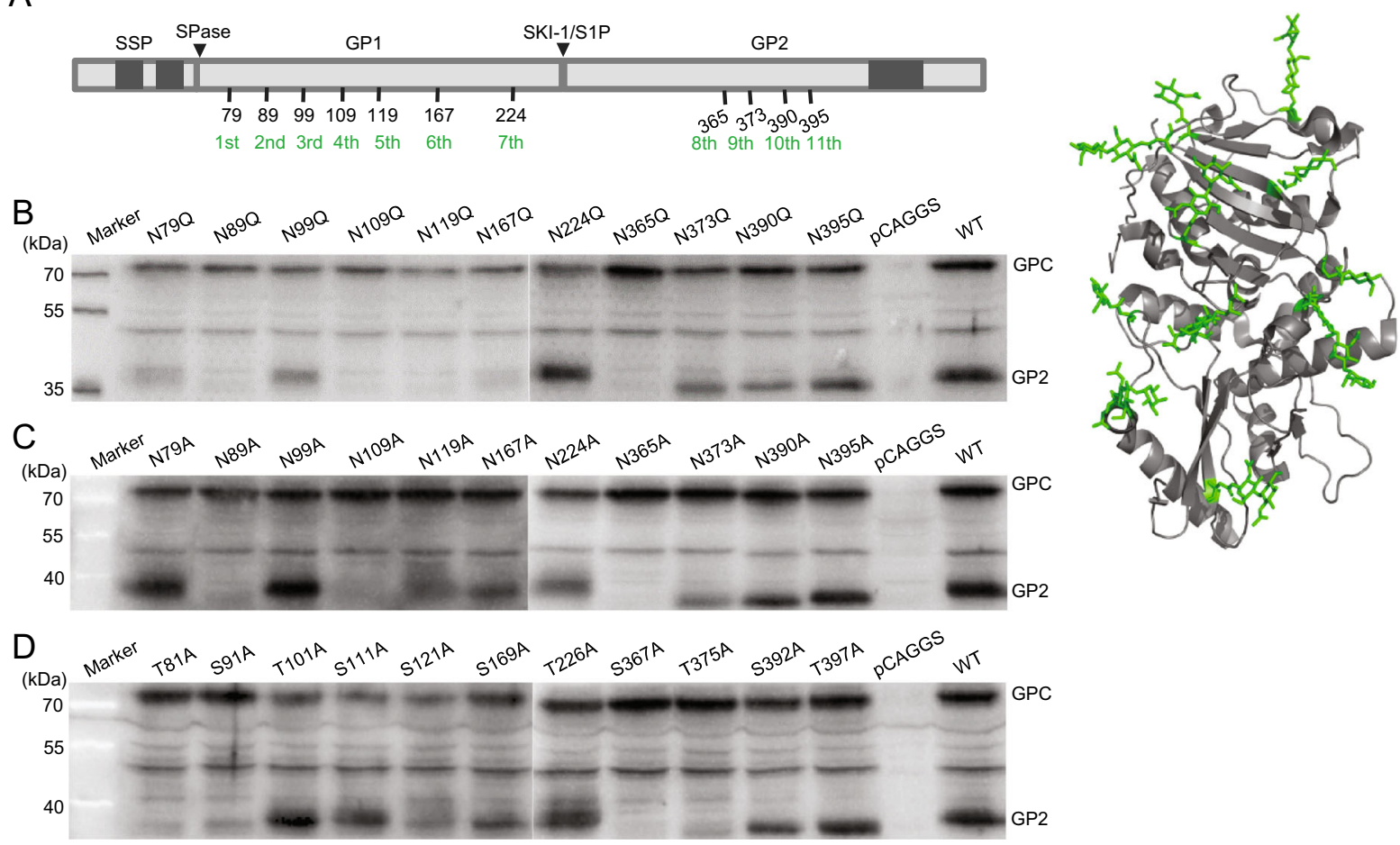

Fig. 1 Proteolytic cleavage of the individual N-linked glycosylation motif mutants. A Schematic diagram and cartoon representation (PDB: 5VK2 (1)) of the glycosylation of LASV GPC. The precursor glycoprotein GPC was cleaved by SPase and SKI-1/S1P. The mature GPC contains SSP (1-58), GP1 (59-259), and GP2 (260-491) domains and is modified by $11 \mathrm{~N}$-linked glycan chains (green).
Transmembrane domains are indicated in gray. B-D HEK 293 T cells expressing the wild-type GPC and individual $N$-glycosylation mutants of $\mathrm{N} \rightarrow \mathrm{Q}(\mathbf{B}), \mathrm{N} \rightarrow \mathrm{A}(\mathbf{C})$, and $\mathrm{S} / \mathrm{T} \rightarrow \mathrm{A}(\mathbf{D})$, respectively. The expressed proteins were separated via SDS-PAGE, and western blotting was carried out using anti-GP2 antisera. Images chosen are representative of at least three independent assays. 
cells were infected with the WT and mutant pseudotyped viruses with the same genome copies.

To generate individual glycan deletion mutants, we introduced three amino acid substitutions $(\mathrm{N} \rightarrow \mathrm{A}$, $\mathrm{N} \rightarrow \mathrm{Q}$, and $\mathrm{T} / \mathrm{S} \rightarrow \mathrm{A}$ ) into the $11 \mathrm{~N}$-glycosylation motifs using 33 pairs of primers (Supplementary Table S1) (Synthesized by Sangon, Shanghai, China). The recombinant plasmids underwent PCR amplification to perform site-directed mutagenesis, after which the template was removed by $D p n I$ restriction digestion, and products were obtained via gel extraction, transformation, and monoclonal identification.

\section{Mice}

Specific pathogen-free (SPF) 6-week-old female BALB/c mice were maintained at the Laboratory Animal Center of Wuhan Institute of Virology, Chinese Academy of Sciences (CAS). All mice were fed in independent ventilated cages (IVCs), and the IVCs were kept within an SPF barrier environment for experimental animals. The feed was sterilized via $\mathrm{Co}^{60}$ irradiation, and water was sterilized using an autoclave.

\section{Immunization Strategy}

Immunization was performed via intramuscular injection using $40 \mu \mathrm{g}$ of each plasmid in the medial thigh of each mouse while avoiding blood vessels. To improve immunogenicity, mice were shocked with an Electro Square Porator (BTX, ECM830) using the cross method with the injection hole as the center. Six mice in each group were immunized three times over a 2-week interval, and at 10 days after the final immunization, mice were euthanized via decapitation; eyeball enucleation was conducted for blood collection.

\section{Separation of Mouse Spleen Lymphocytes}

Lymphocyte separation was performed using an EZ-Sep kit (Dakewe Biotech Co., Ltd., Beijing, China) according to the manufacturer's instructions. Mouse spleen was soaked in Roswell Park Memorial Institute (RPMI) 1640 medium. EZ-Sep separation solution (3-4 $\mathrm{mL}$ ) was then added to a sterile $3 \mathrm{~cm}$ culture dish, over which a nylon mesh was fixed with hemostatic forceps. The spleen was placed onto the mesh for grinding and the grinding solution was rapidly transferred to a $15 \mathrm{~mL}$ centrifuge tube along with $\sim 500 \mu \mathrm{L}$ of serum-free RPMI 1640 along the tube wall; the solution was centrifuged at $800 \times g$ at $25^{\circ} \mathrm{C}$ for $30 \mathrm{~min}$. The lymphocyte layer was transferred to a new $15 \mathrm{~mL}$ centrifuge tube, followed by the addition of $10 \mathrm{~mL}$ serum-free RPMI 1640 medium and centrifugation at $250 \times g$ for $10 \mathrm{~min}$. The supernatant was then carefully removed; the cells were resuspended with $500 \mu \mathrm{L}$ RPMI 1640 medium containing $10 \% \mathrm{FBS}$, and $10 \mu \mathrm{L}$ was used for tenfold serial dilutions for cell counting. The solution was diluted to a cell density of $2 \times 10^{7}$ cells $/ \mathrm{mL}$, transferred to a 96-well U-shapedbottom plate at $100 \mu \mathrm{L} / w e l l$, and cultured in a cell incubator with $5 \% \mathrm{CO}_{2}$ at $37{ }^{\circ} \mathrm{C}$.

\section{Detection of $\mathrm{CD}^{+}{ }^{+}$and $\mathrm{CD8}{ }^{+} \mathrm{T}$ Cells and Cytokines}

The culture system used to stimulate spleen lymphocytes involved the application of a stimulator and co-stimulator $[($ anti-CD28) + Golgi blocker $($ BFA $)+$ spleen lymphocytes $\left(2 \times 10^{6}\right.$ cells) $]$. Negative control, positive control, and experimental groups underwent stimulation with phosphate-buffered saline (PBS), phorbol myristate acetate/ionomycin, and polypeptide (Supplementary Table S2) (Synthesized by Bankpeptide, Heifei, China) (Vita et al. 2019), respectively. After $4.5 \mathrm{~h}$ in culture, the solution was centrifuged at $800 \times g$ for 3 min; $50 \mu \mathrm{L}$ TruStain $\mathrm{FcX}^{\mathrm{TM}}$ (mouse anti-CD16/32; BioLegend, San Diego, CA, USA) was added to reduce non-specific fluorescent staining, incubated at $4{ }^{\circ} \mathrm{C}$ for $10 \mathrm{~min}$, and centrifuged at $800 \times g$ for $3 \mathrm{~min}$. PBS solution was then used to dilute fluorescently labeled antibodies (PE-conjugated rat antimouse CD8a, FITC-conjugated rat anti-mouse CD4, PE$\mathrm{Cy}^{\mathrm{TM}}$ 7-conjugated hamster anti-mouse $\mathrm{CD} 3 \mathrm{e}$ ), and 7-AAD viability staining solution (BioLegend) by 1:200. They were then added $(100 \mu \mathrm{L} /$ well $)$ for staining at $4{ }^{\circ} \mathrm{C}$ for $20 \mathrm{~min}$ in the dark. After washing, the cells were fixed, permeabilized, and subjected to intracellular staining. An allophycocyanin-conjugated rat anti-mouse IFN- $\gamma$ antibody (1:200) was then added and incubated at $4{ }^{\circ} \mathrm{C}$ for $30 \mathrm{~min}$ in the dark. The cells were filtered through a $200 \mu \mathrm{m}$ nylon mesh before being loaded onto the flow cytometer (BD FACSAria III). Cytokines were detected using a cytometric bead array kit (BD Biosciences, Franklin Lakes, NJ, USA) according to the manufacturer's instructions.

\section{Antibody Titration}

The sera were collected from each immunized mouse 10 day after the last immunization to determine specific IgG using cell-based ELISA. The serum was diluted 50-fold, followed by separation into eight gradient dilutions at 1:4 ratios. HeLa cells transfected with the individual glycan deletion GPC or WT GPC were served as antigens, which were blocked, washed, and incubated with serums, followed by detection with HRP-conjugated AffiniPure Goat Anti-Mouse IgG (Proteintech, Wuhan, China). 


\section{Antibody Neutralization}

Serum was diluted tenfold with FBS-free medium and then separated into six gradients at 1:2 ratios. Forty microliters of the diluted serum were mixed with $10 \mu \mathrm{L}$ LASVpv at $37{ }^{\circ} \mathrm{C}$ for $1 \mathrm{~h}$. The mixture was added to Vero cells for $1 \mathrm{~h}$ incubation. Neutralization activities were determined $24 \mathrm{~h}$ later using the Rluc assay system (Promega, Madison, WI, USA).

\section{Statistical Analysis}

All results are expressed as the mean \pm standard error. A two-tailed Student's $t$-test was used to determine statistical significance between groups. All statistical analyses were performed using GraphPad Prism software (ver. 6.0; San Diego, CA, USA). $P<0.05$ was considered statistically significant.

\section{Results}

\section{Effects of N-Glycosylation Modification on GPC Cleavage}

To investigate the influences of different mutations of the 11 glycosylation motifs upon GPC cleavage, the corresponding asparagine residues were individually mutated to glutamine or alanine, or the serine/tyrosine residues in the motif were mutated to alanine. Thus, 33 mutants were constructed, and western blotting was performed using antiserum against GP2. As shown in Fig. 1, all mutants of the $\mathrm{N}$-glycan motifs within GP2 resulted in slight decreases $(\sim 2 \mathrm{kDa})$ in the molecular weight of the respective GP2 bands relative to the wild-type (WT) GP2 band.

We determined the effect of the N-linked glycans on protease cleavage by testing for the presence of cleaved GP2. Disruption of the N-linked glycosylation motif by substitution of asparagine with the structurally similar glutamine on the 2nd, 4th, 5th, or 8th motif (corresponding to N89Q, N109Q, N119Q, N365Q) inhibited proteolytical processing, while substituting glutamine for asparagine at the remaining seven glycosylation motifs (corresponding to N79Q, N99Q, N167Q, N224Q, N373Q, N390Q, N395Q) did not alter GP1-GP2 cleavage (Fig. 1B). Similarly, disruption of the glycosylation motif by substitution of asparagine with alanine on the 2nd, 4th, or 8th motif (corresponding to N89A, N109A, N365A) abolished proteolytical processing, and the mutant in the 5th or 9th (corresponding to N119A, N373A) exerted mild inhibition, whereas the other $\mathrm{N}$ to $\mathrm{A}$ mutants had no influence (Fig. 1C). Intriguingly, substitution of serine at the 8th glycosylation site with alanine (corresponding to S367A) abolished the GP1-GP2 cleavage, and the mutant in the 1st, 2nd, 5th, or 9th (corresponding to T81A, S91A, S121A, T375A) exerted mild inhibition on the cleavage efficiency (Fig. 1D). It was shown that disrupting the glycosylation motifs by introducing different mutants led to differing results, which might be due to the changes of the residue per se, rather than the loss of the specific glycan. However, all three mutants led to a decrease in cleavage efficiency on the 2nd (N89), 5th (N119), or 8th (N365) glycosylation motif, suggesting that the 2nd, 5th, or 8th N-linked glycan was indispensable for GP1-GP2 cleavage. Given the consistency in the results for the $\mathrm{N}$ to $\mathrm{A}$ and $\mathrm{N}$ to $\mathrm{Q}$ substitutions, and that the structures of $\mathrm{N}$ and $\mathrm{Q}$ are the most similar, we used the $\mathrm{N}$ to $\mathrm{Q}$ mutant for further research, which caused a minimal change to the spatial structure of LASV GPC while ensuring removal of the glycosylation-site motif.

\section{Effects of N-Glycosylation Modification on the Infectivity of LASV Pseudotype Virus}

To evaluate the influence of the individual glycan on the pseudotype virus infectivity, we produced VSV particles bearing the altered LASV GP proteins. The transduction activities were evaluated in Vero cells (Fig. 2). Disruption of the N-linked glycan by the $\mathrm{N}$ to $\mathrm{Q}$ substitution on the 2nd, 4th, 5th, or 8th glycosylation motif (corresponding to N89Q, N109Q, N119Q, N365Q) led to a significant loss of LASVpv (LASV pseudovirus) entry when compared with LASVpv packaged with WT GPC. Deletion of the 2nd or 8th N-linked glycan (corresponding to N89Q, N365Q) completely inhibited the infectivity, and deletion of the 4th

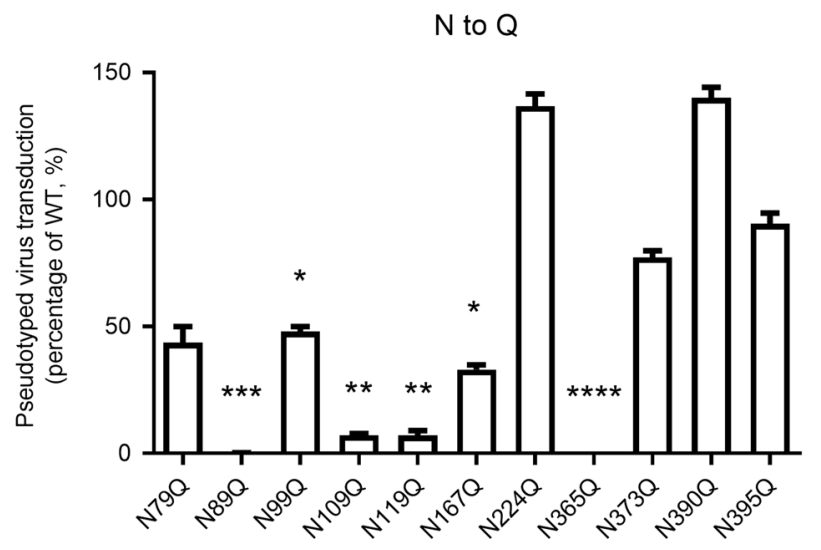

Fig. 2 The transduction activity of pseudotyped viruses bearing mutant GPC. The genome copies of the pseudotype viruses bearing the WT and mutant GPC were quantified using qPCR. Vero cells were infected with the WT and mutant viruses with the same genome copies and the Rluc were determined $24 \mathrm{~h}$ later. Data are presented as mean $\pm \mathrm{SD}$ of at least three independent assays. $* P<0.05$, $* * P<0.01$, *** $P<0.001, * * * * P<0.0001$, compared to WT. 
or 5th N-linked glycan (corresponding to N109Q, N119Q) led to partial inhibition. These results were in line with the protease cleavage results depicted above, indicating that efficient cleavage of premature GPC was a prerequisite for downstream function.

\section{Effects of N-Glycosylation Modification on Effector $\mathrm{CD}^{+}{ }^{+} \mathrm{T}$ Cells and $\mathrm{CD}^{+}{ }^{+} \mathrm{T}$ Cells among Spleen Lymphocytes}

The $11 \mathrm{~N}$-linked glycans on LASV GP play an important role in GPC cleavage and maturation, as well as in pseudovirus infection. Additionally, glycan chain is involved in various aspects of the immune response (Bowden et al. 2009; Abraham et al. 2010; Cohen-Dvashi et al. 2015; Sommerstein et al. 2015; Watanabe et al. 2018). Therefore, we focused on how modification of the $11 \mathrm{~N}$-linked glycans on LASV GP influences the immune response to LASV GPC. We employed recombinant GPC plasmids with $\mathrm{N}$ to $\mathrm{Q}$ substitution as the DNA vaccine used to immunize $\mathrm{BALB} / \mathrm{c}$ mice. $\mathrm{BALB} / \mathrm{c}$ mice were immunized with 13 groups (6 mice per group) of DNA vaccines, including 11 groups of mutated recombinant plasmids, pCAGGS-GPC ${ }_{\mathrm{N} \rightarrow \mathrm{Q}}$, WT GPC, and control pCAGGS. Immunofluorescence analyses of CD3, CD4, CD8, and interferon (IFN) $\gamma$ were performed (Fig. 3). The proportions of $\mathrm{CD}^{+} \mathrm{T}$ cells in spleen lymphocytes in $78 \mathrm{BALB} / \mathrm{c}$ mice across the 13 vaccinated groups were within the range ( $\sim 40 \%)$ of those observed during normal immune response, whereas the deletion of the 1st, $2 \mathrm{nd}$, 4th, or 8 th (N79Q, N89Q, N109Q, N365Q) glycan caused the proportion of $\mathrm{CD}^{+} \mathrm{T}$ cells to decrease slightly when compared to the WT (Fig. 3A). Moreover, abolishment of the 1st, 2nd, or 6th (N79Q, N89Q, N167Q) glycan caused the proportion of $\mathrm{CD}^{+} \mathrm{T}$ cells to decrease, whereas abolishment of the 5th, 9th, or 10th (N119Q, N373Q, N390Q) glycan led to the proportion of $\mathrm{CD}^{+} \mathrm{T}$ cells to increase (Fig. 3B, 3C).

Lymphocytes that secrete IFN- $\gamma$ represent an effector subset of these cells. First, we found that immunization with the WT resulted in a significant increase in the proportion of both the effector $\mathrm{CD} 4^{+}$and $\mathrm{CD}^{+}$cells relative to the control plasmid vaccine group. Moreover, deletion of the 1st, 3rd, 5th or 6th (N79Q, N99Q, N119Q, N167Q) $\mathrm{N}$-linked glycan on GPC significantly enhanced the proportion of effector $\mathrm{CD}^{+}$cells, and deletion of the remaining seven $\mathrm{N}$-sugar chains had no effect on their proportion. For $\mathrm{CD}^{+}$effector $\mathrm{T}$ cells, deletion of the $1 \mathrm{st}$, 2nd, 3rd, 4th 5th, 6th, 9th (N79Q, N89Q, N99Q, N109Q, N119Q, N167Q, N373Q) N-linked glycan enhanced their proportion relative to IFN- $\gamma$, and deletion of the 7 th, 8 th, 10th, or 11th (N224Q, N365Q, N390Q, N395Q) N-linked glycan had no effect (Fig. 3D, 3E).
A

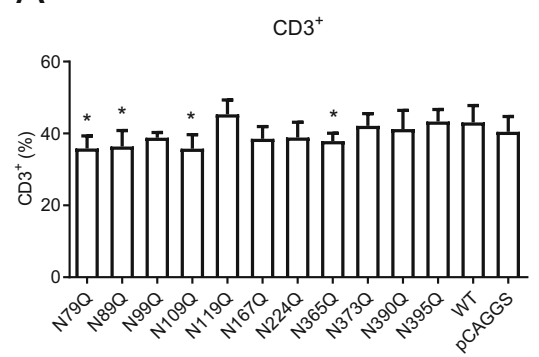

D

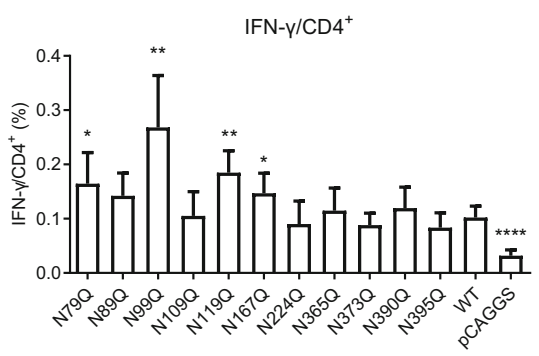

B

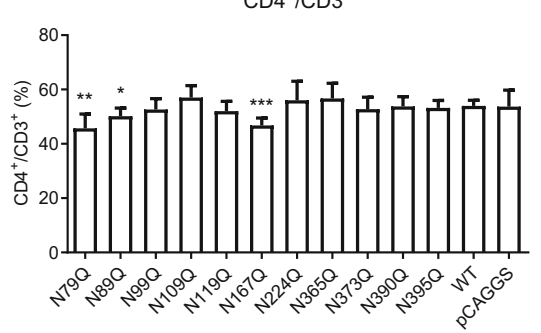

E

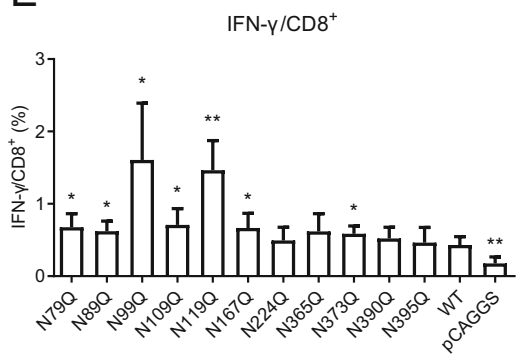

C

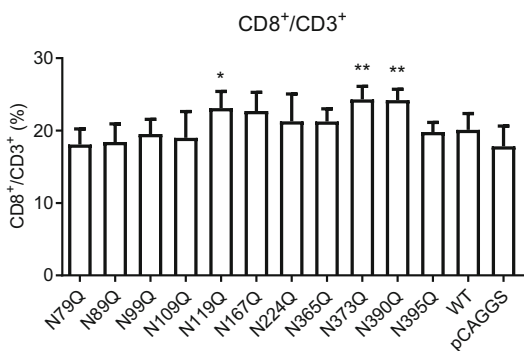

Fig. 3 Effects of $N$-glycan deletion on T cells in spleen lymphocytes. The mouse spleen lymphocytes were separated, cultured, and stained with phycoerythrin (PE)-conjugated rat anti-mouse CD8a, fluorescein isothiocyanate-conjugated rat anti-mouse CD4, PE-CyTM7-conjugated hamster anti-mouse $\mathrm{CD} 3 \mathrm{e}$, and $7-\mathrm{AAD}$ viability staining solution (BioLegend) at $4{ }^{\circ} \mathrm{C}$ for $20 \mathrm{~min}$ in the dark. A-C Effects of individual $\mathrm{N}$-glycosylation deletions on $\mathrm{CD}^{+}(\mathbf{A}), \mathrm{CD}^{+}(\mathbf{B})$, and $\mathrm{CD}^{+}(\mathbf{C}) \mathrm{T}$ cells in spleen lymphocytes. Effector $\mathrm{T}$ cells $\mathrm{CD} 4^{+}$ (D) and $\mathrm{CD}^{+}(\mathbf{E}) \mathrm{T}$ cells were stained with allophycocyaninconjugated rat anti-mouse IFN-y antibody (1:200). Each group contained five to six mice. $* P<0.05$, $* * P<0.01$, *** $P<0.001$, $* * * * P<0.0001$, compared to $\mathrm{WT}$. 


\section{Effects of N-Linked Glycans on Cytokines Secreted by Spleen Lymphocytes}

Among the seven cytokines tested, interleukin (IL)-2, IFN- $\gamma$, and tumor necrosis factor (TNF)- $\alpha$ are associated with the Th1-type cellular immune response, IL-4, IL-6, and IL-10 with the Th2-type response, and IL-17A to the Th17-type response. Figure 4 shows that all seven cytokines, except for IL-17A, increased in WT group relative to control pCAGGS group. Specifically, deletion of the individual 3rd (N99Q) to 10th (N390Q) N-linked glycan on GPC significantly increased IL-2 levels in spleen lymphocytes relative to those in mice receiving WT GPC (Fig. 4A), whereas deletion of the individual 3rd, 5th, 6th, 8th, 9th, or 10th (N99Q, N119Q, N167Q, N365Q, N373Q, N390Q) glycan increased IFN- $\gamma$ levels (Fig. 4B); TNF- $\alpha$ was only slightly elevated when either the 3rd (N99Q) or 9th (N373Q) glycan was abolished (Fig. 4C).
Furthermore, we found that deletion of the 1st (N79Q) or 5th (N119Q) N-linked glycan on GPC significantly increased IL-4 secretion, deletion of the 2nd, 3rd, 4th, or 8th (N89Q, N99Q, N109Q, N365Q) slightly increased IL-4 secretion, and deletion of the remaining five glycans had no effect (Fig. 4D). However, IL-6 levels in either glycan mutant motif showed no significant difference relative to levels in the WT group (Fig. 4E). Furthermore, deletion of the 3rd, 5th, 7th, 8th, 9th, or 10th (N99Q, N119Q, N224Q, N365Q, N373Q, N390Q) N-linked glycan slightly increased IL-10 levels, whereas deletion of the remaining five N-linked glycans had no effect (Fig. 4F).

When studying induction of the Th17-type immune response (Fig. 4G), we observed elevated levels of IL-17A in spleen lymphocytes from mice in the 7th, 8th, or 10th (N224Q, N365Q, N390Q) deletion mutant group relative to those in mice receiving the WT vaccine.

These results indicated that deletion of $\mathrm{N}$-linked glycan on GPC, especially those at the 3rd, 5th, 8th, or 9th site
A
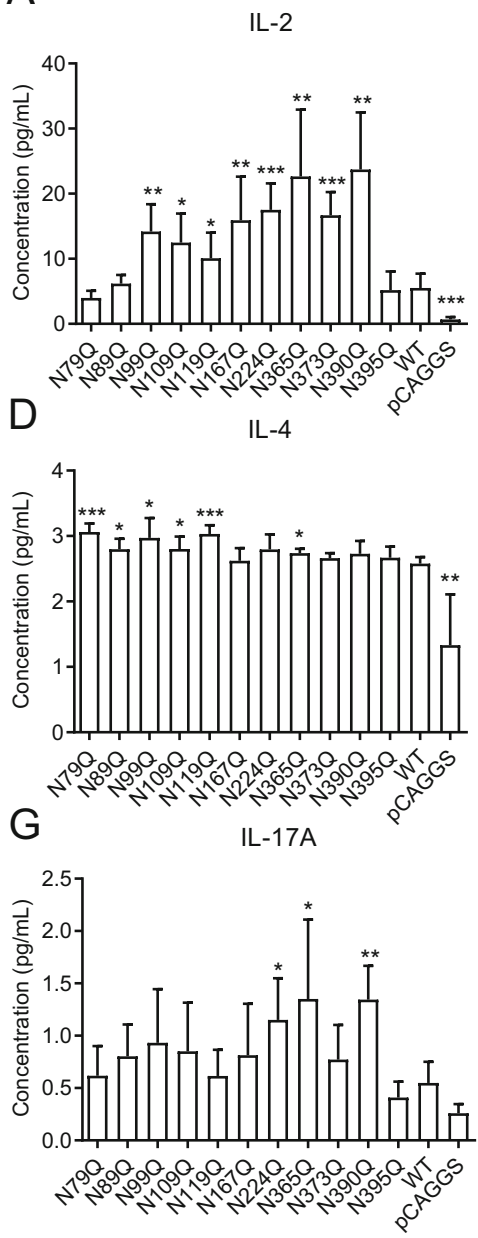

Fig. 4 Effects of $N$-glycan deletions on cytokines secreted by spleen lymphocytes were labeled by using a cytometric bead array kit (BD Biosciences), and IL-2 (A), IFN- $\gamma(\mathbf{B})$, TNF- $\alpha(\mathbf{C})$, IL-4 (D), IL-6 (E),
B
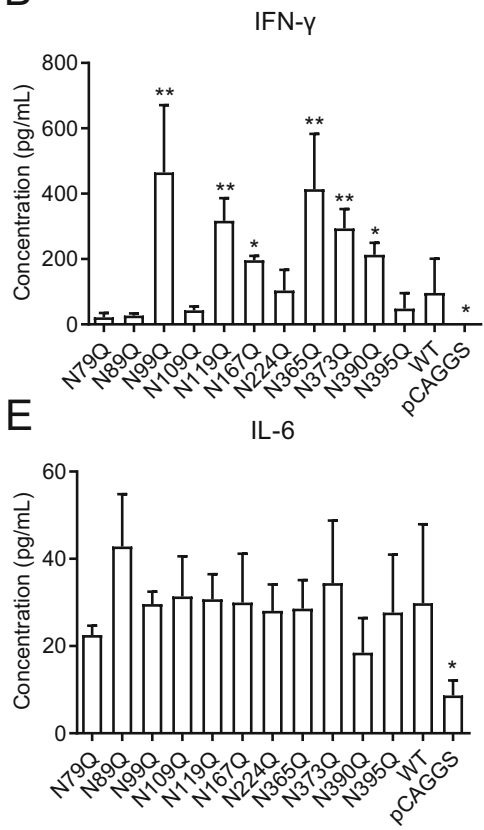

C
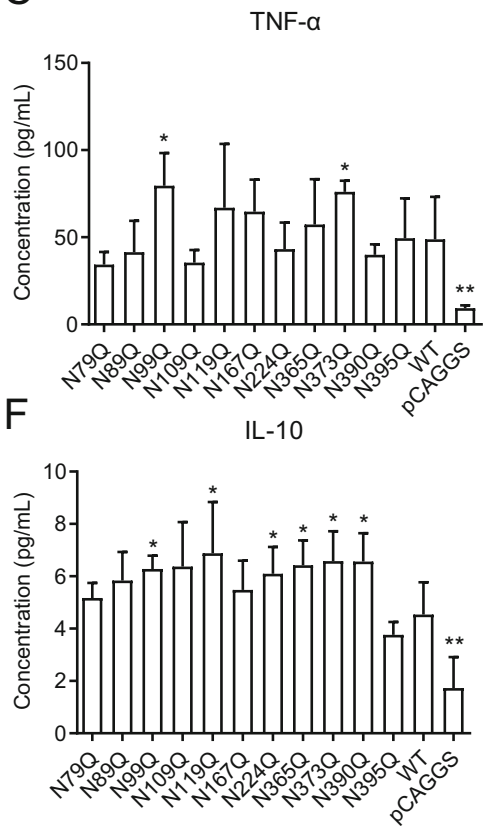

IL-10 (F), and IL-17A (G) were determined using flow cytometry. Each group contained five to six mice. $* P<0.05$, $* * P<0.01$, $* * * P<0.001$, compared to WT. 
(N99Q, N119Q, N365Q, N373Q), primarily enhanced the induction of both Th1 and Th2 immune response. Intriguingly, deletion of the 11th (N395Q) glycan on GPC had no effect on Th1, Th2, and Th17A immune response. These findings suggest these specific glycan chains assist LASV in escaping immune response by reducing host recognition of GPC and precluding induction of the immune response.

\section{Effects of N-Glycosylation Modification on Antibody Titers}

We then detected antibody titers obtained from the individual glycan deletion mutant immunized serum against each mutated GPC variant using a cell-based ELISA.
Figure 5 shows that antibody response to $\mathrm{N}$-glycan deletion GPC showed higher titer to mutant GPC than to WT GPC, suggesting that the glycans on LASV GPC might play a role in immune escape by shielding the epitope, thus making it inaccessible to antibodies. Evaluation of which $\mathrm{N}$-glycosylation site affected antibody titers revealed that deletion of N-linked glycan at the 3rd, 5th, 6th, 8th, or 9th site (N99Q, N119Q, N167Q, N365Q, N373Q) significantly increased the antibody titer. The titers obtained from these five groups could reach to approximately $17(-\log 2)$ whereas the titers from the remaining seven groups were approximately $15(-\log 2) \quad$ (Fig. 5A-5L). Similarly, to compare the antibody titers when titrated with WT GPC, we found that deletion of the 3rd, 5th, 6th, 8th, or 9th
A

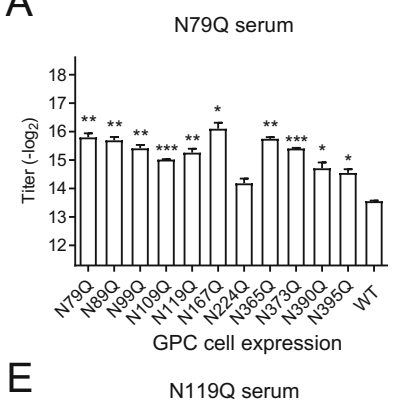

$\mathrm{E}$

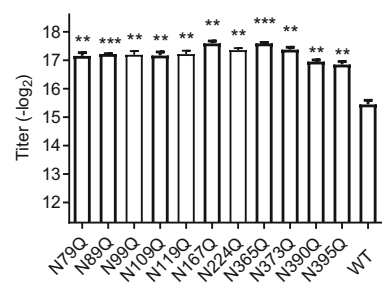

GPC cell expression

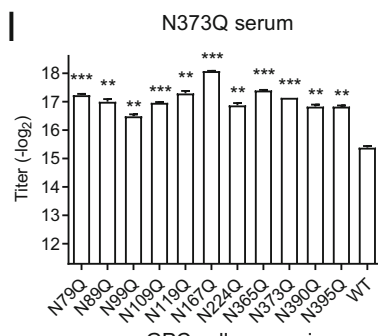

M

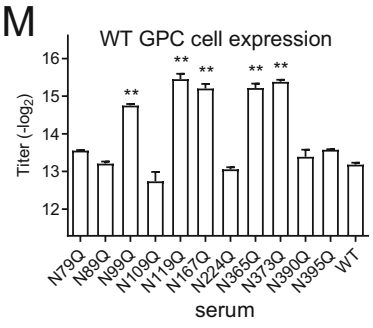

B

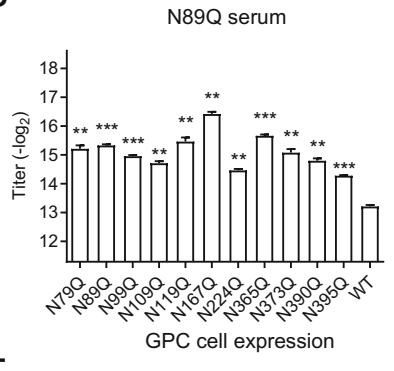

$\mathrm{F}$

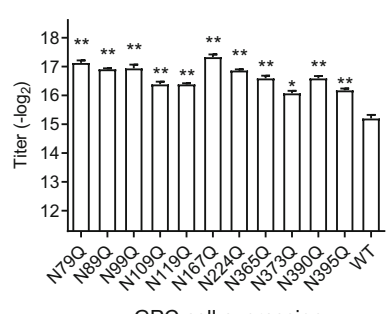

$J$

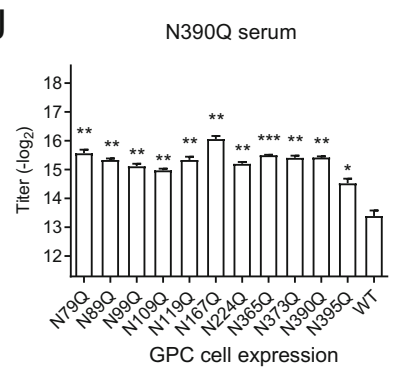

C
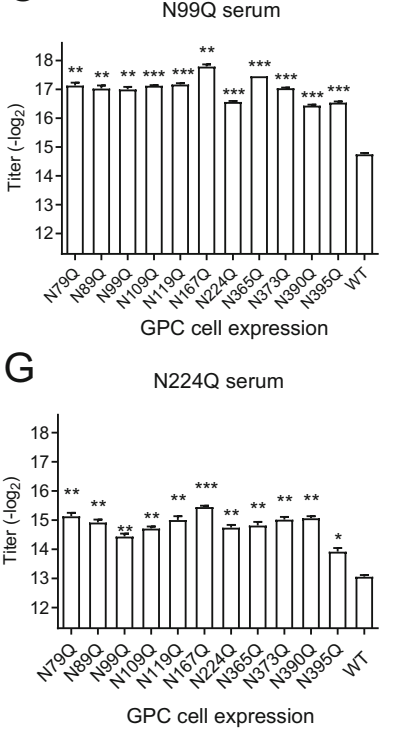

K

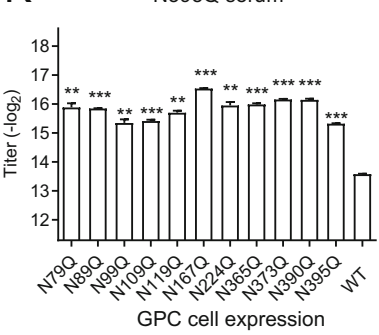

$\mathrm{D}$

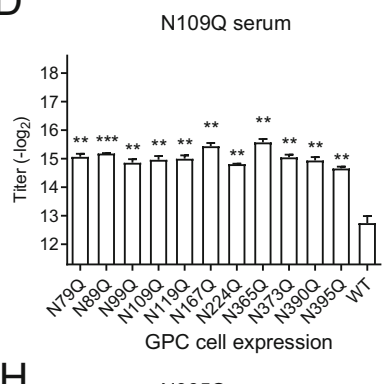

$\mathrm{H}$

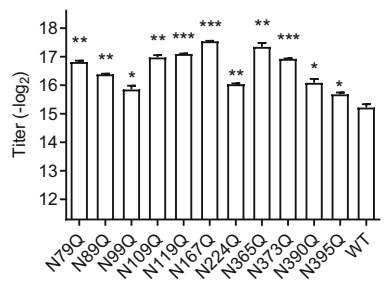

GPC cell expression

$\mathrm{L}$

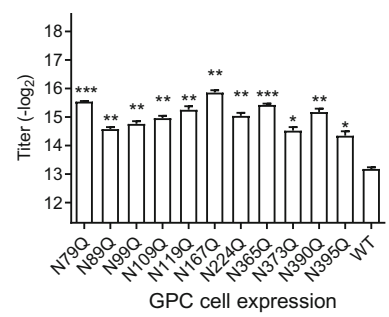

Fig. 5 Antibody response to $N$-glycan deletion GPC showed higher titer to mutant GPC than to WT GPC. After three rounds of immunization, sera were collected, and the titers were determined using cell-based ELISA. A-L The titer of the indicated serum to the mutated GPC. M The titer of the indicated serum to WT GPC. ${ }^{*} P<0.05, * * P<0.01,{ }^{* * *} P<0.001$, compared to WT. 
(N99Q, N119Q, N167Q, N365Q, N373Q) N-linked glycan generated significantly higher antibody titers than other deletion mutants as well as WT (Fig. 5M), thereby indicating a role for the 3rd (N99), 5th (N119), 6th (N167), 8th (N365), and 9th (N373)N-glycosylation sites in the GPC in immune escape and reduced humoral immune response by shielding key GPC epitopes.

\section{Effects of N-Glycosylation Modification on Antibody Neutralization}

We then evaluated the neutralization ability of the serum against LASVpv infection (Fig. 6). Unfortunately, we found that the serum generated by both the WT GPC immunized group and each of the N-linked glycan deletion mutated variants showed minimal to no neutralizing effect to the LASVpv infection.

\section{Discussion}

In a variety of virions such as influenza A virus, human immunodeficiency virus, Ebola virus, etc., a glycan shield on the glycoprotein plays critical roles in the host immune response. The LASV GPC harbors $11 \mathrm{~N}$-linked glycans that almost completely encapsulate the GPC. Therefore, modification of these chains represents a possible strategy for identifying the LASV immune-escape mechanism, given that these chains might shield key epitopes on the protein surface from immune recognition. We hypothesized that removal of these chains could expose the epitopes, thereby promoting host recognition and triggering of the immune response.

First, to investigate the role of individual N-linked glycan in GPC expression and function, we found that using three different strategies to abolish the glycosylation motif, the deletion of the 2nd (N89) or the 8th (N365) glycan led to decreased GPC cleavage and pseudotype virus infectivity. These results were in line with previous reports that commented on the importance of these two glycans in LCMV GPC function (Bonhomme et al. 2011, 2013). As the 2nd (N89) and 8th (N365) glycans are conserved in Mammarenavirus GPC, it was supposed that both of the glycan chains play essential roles in GPC expression and function (Abraham et al. 2010; Hastie et al. 2017). The 2nd (N89) glycan was reported to interact with H92 in the prefusion conformation, and during endocytosis, the glycan chain was rotated and H92 was released, which could bind to the second receptor LAMP1 (Cohen-Dvashi et al. 2015; Hastie et al. 2017). Similarly, the 2nd (N89) glycan of the New World Mammarenavirus Machupo virus was reported to form a stacking interaction with F98, which was essential for receptor binding (Abraham et al. 2010; Bonhomme et al. 2013). The 8th (N365) glycan of LASV GPC was the first glycan in GP2. This glycan was reported to interact with Q232 and R235, shielding the fusion peptide at the tip of GP2, and thus contributing to the stability of the prefusion GPC (Hastie et al. 2017).

It was recently reported that the 3rd (N99) and 5th (N119) glycans on LASV GPC shield the neutralizing epitopes of the virus (Sommerstein et al. 2015; Robinson et al. 2016), and the 5th (N119), 8th (N365) and 9th (N373) glycosylation motifs were reported to be located in the epitopes of GPC (Robinson et al. 2016). Most recently, the 10th (N390) and 11th (N395) glycans were reported to occlude the conformational GPC-B epitope located at the stalk of GPC (Hastie et al. 2019). Notably, evidence from human survivor and vaccine development studies have shown that adaptive immune protection in LASV infection is probably conferred mainly by a cell-mediated immune response, especially for Type I IFN (Fisher-Hoch et al. 2000; ter Meulen et al. 2004; Geisbert et al. 2005; Botten et al. 2006; Baize et al. 2009; Mire et al. 2017; Warner et al. 2018; Ibukun 2020). It was supposed that deletion of the glycan chain would expose the epitope and thus increase the immunogenicity of GPC. We found that deletion of specific $\mathrm{N}$-linked glycan residues on the GPC had no effect on the proportions of lymphocytes $\left(\mathrm{CD} 3^{+}, \mathrm{CD} 4^{+} / \mathrm{CD}^{+}\right.$, and $\left.\mathrm{CD} 8^{+} / \mathrm{CD}^{+}\right)$but significantly increased proportions of effector lymphocytes $\left(\mathrm{IFN}-\gamma^{+} /\right.$ $\mathrm{CD}^{+}$and IFN $-\gamma^{+} / \mathrm{CD} 8^{+}$), especially following deletion of glycan chain at the 1st, 3rd, 5th, or 6th glycosylation site (N79Q, N99Q, N119Q, N167Q). Additionally, we observed significant increases in the secretion of molecules involved in Th1 immune response (IL-2 and IFN- $\gamma$ ) following deletion
Fig. $6 \mathrm{~N}$-glycan deletions showed little effect in improving the neutralization ability of the antibody from sera against the seven individual glycan deletions in GP1 (A) and four in GP2 (B).
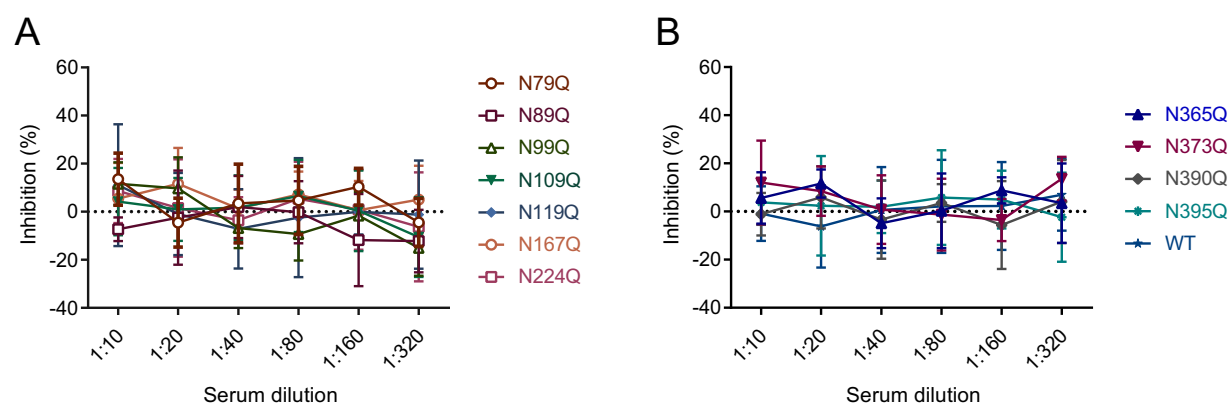
of chain at the 3rd, 5th, 8th, 9th, or 10th glycosylation site (N99Q, N119Q, N365Q, N373Q, N390Q), although this did not affect the secretion of Th2- and Th17-related cytokines. These results support the role of $\mathrm{N}$-linked glycans in inhibiting host recognition and Th1 immune response.

Additionally, analysis of changes in antibody titers from the sera of mice immunized with GPC variants revealed general increases in titer following glycan removal, suggesting that the presence of these glycan chains promotes immune escape by shielding the antigenic epitope. Moreover, we verified that the antibodies generated by all GPC variants showed no neutralizing effect on the WT LASVpv. This indicated that the epitopes exposed by the deletion of $\mathrm{N}$-linked glycans on the GPC did not generate neutralizing antibodies, or the glycan chains shield the neutralizing epitopes of the WT virus.

In summary, we found that the 3rd, 5th, 6th, 8 th, and 9th N-linked glycans (N99, N119, N167, N365, N373) likely shield epitopes on the LASV GPC that reduce host cellular and humoral immune responses. These sites can potentially be used as breakthrough points to develop effective therapeutic or prophylactic antibodies against Lassa fever.

Acknowledgements We thank the Center for Instrumental Analysis and Metrology, Core Facility and Technical Support, and Center for Animal Experiment, Wuhan Institute of Virology, for providing technical assistance. This work was supported by the National Key Research and Development Program of China (2018YFA0507204), the National Natural Sciences Foundation of China (31670165), Wuhan National Biosafety Laboratory, Chinese Academy of Sciences Advanced Customer Cultivation Project (2019ACCP-MS03), the Open Research Fund Program of the State Key Laboratory of Virology of China (2018IOV001).

Author Contributions XZ, GX, and WW conceived and designed the study. Experiments were performed by XZ, YL, JG, JC, ZW, and WW. Data were analyzed by XZ, YL, and WW. XZ and WW wrote the manuscript. All authors read and approved the manuscript.

\section{Compliance with Ethical Standards}

Conflict of interest The authors declare that there are no conflicts of interest.

Animal and Human Rights Statement All mouse studies were performed according to the Regulations of the Administration of Affairs Concerning Experimental Animals in China (WIVA25201801), and the protocols were reviewed and approved by the Laboratory Animal Care and Use Committee at the Wuhan Institute of Virology, CAS.

\section{References}

Abraham J, Corbett KD, Farzan M, Choe H, Harrison SC (2010) Structural basis for receptor recognition by New World hemorrhagic fever arenaviruses. Nat Struct Mol Biol 17:438-444
Baize S, Marianneau P, Loth P, Reynard S, Journeaux A, Chevallier M, Tordo N, Deubel V, Contamin H (2009) Early and strong immune responses are associated with control of viral replication and recovery in lassa virus-infected cynomolgus monkeys. J Virol 83:5890-5903

Bonhomme CJ, Capul AA, Lauron EJ, Bederka LH, Knopp KA, Buchmeier MJ (2011) Glycosylation modulates arenavirus glycoprotein expression and function. Virology 409:223-233

Bonhomme CJ, Knopp KA, Bederka LH, Angelini MM, Buchmeier MJ (2013) LCMV glycosylation modulates viral fitness and cell tropism. PLoS ONE 8:e53273

Botten J, Alexander J, Pasquetto V, Sidney J, Barrowman P, Ting J, Peters B, Southwood S, Stewart B, Rodriguez-Carreno MP, Mothe B, Whitton JL, Sette A, Buchmeier MJ (2006) Identification of protective Lassa virus epitopes that are restricted by HLA-A2. J Virol 80:8351-8361

Bowden TA, Crispin M, Graham SC, Harvey DJ, Grimes JM, Jones EY, Stuart DI (2009) Unusual molecular architecture of the machupo virus attachment glycoprotein. J Virol 83:8259-8265

Cohen-Dvashi H, Cohen N, Israeli H, Diskin R (2015) Molecular mechanism for LAMP1 recognition by Lassa virus. J Virol 89:7584-7592

Eichler R, Lenz O, Garten W, Strecker T (2006) The role of single $\mathrm{N}$-glycans in proteolytic processing and cell surface transport of the Lassa virus glycoprotein GP-C. Virol J 3:41

Fisher-Hoch SP, Hutwagner L, Brown B, McCormick JB (2000) Effective vaccine for lassa fever. J Virol 74:6777-6783

Geisbert TW, Jones S, Fritz EA, Shurtleff AC, Geisbert JB, Liebscher R, Grolla A, Stroher U, Fernando L, Daddario KM, Guttieri MC, Mothe BR, Larsen T, Hensley LE, Jahrling PB, Feldmann H (2005) Development of a new vaccine for the prevention of Lassa fever. PLoS Med 2:e183

Gunther S, Emmerich P, Laue T, Kuhle O, Asper M, Jung A, Grewing $\mathrm{T}$, ter Meulen J, Schmitz H (2000) Imported lassa fever in Germany: molecular characterization of a new Lassa virus strain. Emerg Infect Dis 6:466-476

Hastie KM, Zandonatti MA, Kleinfelter LM, Heinrich ML, Rowland MM, Chandran K, Branco LM, Robinson JE, Garry RF, Saphire EO (2017) Structural basis for antibody-mediated neutralization of Lassa virus. Science 356:923-928

Hastie KM, Cross RW, Harkins SS, Zandonatti MA, Koval AP, Heinrich ML, Rowland MM, Robinson JE, Geisbert TW, Garry RF, Branco LM, Saphire EO (2019) Convergent structures illuminate features for Germline antibody binding and pan-Lassa virus neutralization. Cell 178:e1014

Houlihan C, Behrens R (2017) Lassa fever. BMJ 358:j2986

Ibukun FI (2020) Inter-lineage variation of Lassa virus glycoprotein epitopes: a challenge to Lassa virus vaccine development. Viruses 12:386

Johnson KM, McCormick JB, Webb PA, Smith ES, Elliott LH, King IJ (1987) Clinical virology of Lassa fever in hospitalized patients. J Infect Dis 155:456-464

McCormick JB, King IJ, Webb PA, Scribner CL, Craven RB, Johnson KM, Elliott LH, Belmont-Williams R (1986) Lassa fever. Effective therapy with ribavirin. N Engl J Med 314:20-26

Mire CE, Cross RW, Geisbert JB, Borisevich V, Agans KN, Deer DJ, Heinrich ML, Rowland MM, Goba A, Momoh M, Boisen ML, Grant DS, Fullah M, Khan SH, Fenton KA, Robinson JE, Branco LM, Garry RF, Geisbert TW (2017) Human-monoclonalantibody therapy protects nonhuman primates against advanced Lassa fever. Nat Med 23:1146-1149

Robinson JE, Hastie KM, Cross RW, Yenni RE, Elliott DH, Rouelle JA, Kannadka CB, Smira AA, Garry CE, Bradley BT, Yu H, Shaffer JG, Boisen ML, Hartnett JN, Zandonatti MA, Rowland MM, Heinrich ML, Martinez-Sobrido L, Cheng B, de la Torre JC, Andersen KG, Goba A, Momoh M, Fullah M, Gbakie M, 
Kanneh L, Koroma VJ, Fonnie R, Jalloh SC, Kargbo B, Vandi MA, Gbetuwa M, Ikponmwosa O, Asogun DA, Okokhere PO, Follarin OA, Schieffelin JS, Pitts KR, Geisbert JB, Kulakoski PC, Wilson RB, Happi CT, Sabeti PC, Gevao SM, Khan SH, Grant DS, Geisbert TW, Saphire EO, Branco LM, Garry RF (2016) Most neutralizing human monoclonal antibodies target novel epitopes requiring both Lassa virus glycoprotein subunits. Nat Commun 7:11544

Sommerstein R, Flatz L, Remy MM, Malinge P, Magistrelli G, Fischer N, Sahin M, Bergthaler A, Igonet S, Ter Meulen J, Rigo D, Meda P, Rabah N, Coutard B, Bowden TA, Lambert PH, Siegrist CA, Pinschewer DD (2015) Arenavirus glycan shield promotes neutralizing antibody evasion and protracted infection. PLoS Pathog 11:e1005276

ter Meulen J, Badusche M, Kuhnt K, Doetze A, Satoguina J, Marti T, Loeliger C, Koulemou K, Koivogui L, Schmitz H, Fleischer B, Hoerauf A (2000) Characterization of human CD4(+) T-cell clones recognizing conserved and variable epitopes of the Lassa virus nucleoprotein. J Virol 74:2186-2192

ter Meulen J, Badusche M, Satoguina J, Strecker T, Lenz O, Loeliger C, Sakho M, Koulemou K, Koivogui L, Hoerauf A (2004) Old and New World arenaviruses share a highly conserved epitope in the fusion domain of the glycoprotein 2, which is recognized by Lassa virus-specific human CD4+ T-cell clones. Virology 321:134-143

Vita R, Mahajan S, Overton JA, Dhanda SK, Martini S, Cantrell JR, Wheeler DK, Sette A, Peters B (2019) The immune epitope database (IEDB): 2018 update. Nucl Acids Res 47:D339-D343

Wang P, Liu Y, Zhang G, Wang S, Guo J, Cao J, Jia X, Zhang L, Xiao G, Wang W (2018) Screening and identification of Lassa virus entry inhibitors from an FDA-approved drugs library. J Virol 92:e00954-e1918

Warner BM, Safronetz D, Stein DR (2018) Current research for a vaccine against Lassa hemorrhagic fever virus. Drug Des Devel Ther 12:2519-2527

Watanabe Y, Raghwani J, Allen JD, Seabright GE, Li S, Moser F, Huiskonen JT, Strecker T, Bowden TA, Crispin M (2018) Structure of the Lassa virus glycan shield provides a model for immunological resistance. Proc Natl Acad Sci USA 115:7320-7325 